\title{
Finding Feasible Counter-examples when Model Checking Abstracted Java Programs*
}

\author{
Corina S. Păsăreanu ${ }^{1}$, Matthew B. Dwyer ${ }^{1}$, and Willem Visser ${ }^{2}$ \\ 1 Department of Computing and Information Sciences, Kansas State University, USA \\ 2 RIACS, NASA Ames Research Center, Moffett Field, USA \\ pcorina@cis.ksu.edu
}

\begin{abstract}
Despite recent advances in model checking and in adapting model checking techniques to software, the state explosion problem remains a major hurdle in applying model checking to software. Recent work in automated program abstraction has shown promise as a means of scaling model checking to larger systems. Most common abstraction techniques compute an upper approximation of the original program. Thus, when a specification is found true for the abstracted program, it is known to be true for the original program. Finding a specification to be false, however, is inconclusive since the specification may be violated on a behavior in the abstracted program which is not present in the original program. We have extended an explicit-state model checker, Java PathFinder (JPF), to analyze counter-examples in the presence of abstractions. We enhanced JPF to search for "feasible" (i.e. nondeterminismfree) counter-examples "on-the-fly", during model checking. Alternatively, an abstract counter-example can be used to guide the simulation of the concrete computation and thereby check feasibility of the counterexample. We demonstrate the effectiveness of these techniques on counterexamples from checks of several multi-threaded Java programs.
\end{abstract}

\section{Introduction}

In the past decade, model checking has matured into an effective technique for reasoning about realistic components of hardware systems and communication protocols. The past several years have witnessed a series of efforts aimed at applying model checking techniques to reason about software implementations (e.g., Java source code 81224 ). While the conceptual basis for applying model checking to software is reasonably well-understood, there are still unsettled questions about whether effective tool support can be constructed that allows for realistic software requirements to be checked of realistic software descriptions in a practical amount of time. Most researchers in model checking believe that propertypreserving abstraction of the state-space will be necessary to make checking

\footnotetext{
* Supported in part by NSF under grants CCR-9703094 and CCR-9708184, by NASA under grant NAG-02-1209, by DARPA/ITO's PCES program through AFRL Contract F33615-00-C-3044, and was performed for the Formal Verification of Integrated Modular Avionics Software Cooperative Agreement, NCC-1-399, sponsored by Honeywell Technology Center and NASA Langley Research Center.
}

T. Margaria and W. Yi (Eds.): TACAS 2001, LNCS 2031, pp. 284-298 2001

(C) Springer-Verlag Berlin Heidelberg 2001 
of realistic systems practical (e.g., 611 19). There are a variety of challenges in bringing this belief to reality. This paper addresses one of those challenges, namely, the problem of automating the analysis of counter-examples that have been produced from abstract model checks in order to determine whether they represent real system defects.

The work described in this paper involves the integration of two recently developed tools for model checking Java source code : Bandera 8 and Java PathFinder \%4. Bandera is a toolset that provides automated support for reducing a program's state space through the application of program slicing and the compilation of abstract definitions of program data types. The resulting reduced Java program is then fed to JPF which performs an optimized explicitstate model check for program properties (e.g., assertion violations or deadlock). If the search is free of violations then the program properties are verified. If a violation is found the situation is less clear. Bandera uses abstractions that preserve the ability to prove all paths properties (e.g., such as assertions or linear temporal logic formulae). To achieve state space reduction, however, the ability to disprove such properties is sacrificed. This means that a check of an abstracted system may fail either because the program has an error or because the abstractions introduce spurious executions into the program that violate the property. The former are of interest to a user, while the latter are a distraction to the user, especially if spurious results occur in large numbers.

Several approaches have been proposed recently for analyzing the feasibility of counter-examples of abstracted transition-system models 5:34. While our work shares much in common with these approaches, it is distinguished from them in four ways: $(i)$ it treats the abstraction a program's data, as well as the run-time system scheduler and the property to be checked, $(i i)$ the feasibility of a counter-example is judged against the semantics of a real programming language (i.e., Java), (iii) we advocate multiple approaches for analyzing feasibility with different cost/precision profiles, and (iv) our work is oriented toward detecting defects in the presence of abstraction. We will demonstrate the practical utility of an implementation of our approaches by applying them to the analysis of counter-examples for several real multi-threaded Java applications.

Safe abstractions often result in program models where the information required to decide conditionals is lost and hence nondeterministic choice needs to be used to encode such conditionals (i.e., to account for both true and false results). Nondeterministic choice is also used to model the possible decisions that a thread (or process) scheduler would make. Such abstractions are safe for all paths properties since they are guaranteed to include all behaviors of the unabstracted system. The difficulty lies in the fact that they may introduce many behaviors that are not possible. To sharpen the precision of the abstract model (by eliminating some spurious behaviors) one minimizes the use of nondeterminism and it can be shown that the absence of nondeterminism equates to feasibility 93. Section describes how program data, the property and scheduler behavior are abstracted in Bandera/JPF using nondeterminism. 
JPF can perform a state-space search that is bounded by nondeterministicchoice operations; a property violation that lies within this space has a counterexample that is free of nondeterminism and is hence feasible. JPF can also perform simulation of the concrete program guided by an abstract counter-example. If a corresponding concrete program trace exists then the counter-example is feasible. Section 4 describes these two techniques for analyzing program counterexamples that were added to JPF. Section 5 describes several defective Java applications whose counter-examples were analyzed using these techniques. In Section 60 we discuss related and future work and we conclude in Section 7 In the next section, we give some brief background on Bandera and JPF.

\section{Background}

Bandera 8 is an integrated collection of program analysis and transformation components that allows users to selectively analyze program properties and to tailor the analysis to that property so as to minimize analysis time. Bandera exploits existing model checkers, such as Spin 16, SMV 20, and JPF 24, to provide state-of-the-art analysis engines for checking program-property correspondence. Bandera provides support for reducing a program's state-space via program slicing 15 and data abstraction.

Data abstraction automates the reduction in size of the data domains over which program variables range 13 . A type inference algorithm is applied to ensure that a consistent set of abstractions are applied to program data. This type-based approach to abstraction is complementary to predicate abstraction approaches that reduce a program by preserving the ability to decide specific user-define predicates; JPF's companion tool implements predicate abstraction programs written in Java 25 .

Java PathFinder is a model checker for Java programs that can check any Java program, since it is built on top of a custom made Java Virtual Machine (JVM), for deadlock and violations of user-defined assertions 24. In JPF special attention is paid to reducing the number of states, rather than execution speed as is typical of commercial JVMs, since this is the major efficiency concern in explicit-state model checking. Users have the ability to set the granularity of atomic steps during model checking to: byte-codes, source lines (the default) or explicit atomic blocks (through calls to beginAtomic() and endAtomic() methods from a special class called Verify). A JPF counter-example indicates how to execute code from the initial state of the program to reach the error. Each step in the execution contains the name of the class the code is from, the file the source code is stored in, the line number of the source file that is currently being executed and the a number identifying the thread that is executing. Using only thread numbers in each step JPF can simulate the erroneous execution.

\section{Program Abstraction}

Given a concrete program and a property, the strategy of verification by using abstraction involves: (i) defining an abstraction mapping that is appropriate for 
the property being verified and using it to transform the concrete program into an abstract program, (ii) transforming the property into an abstract property, (iii) verifying that the abstract program satisfies the abstract property, and finally (iv) inferring that the concrete program satisfies the concrete property. In this section, we summarize foundational issues that underlie these steps.

\subsection{Data Abstraction}

The abstract interpretation (AI) 9 framework as described in a large body of literature establishes a rigorous semantics-based methodology for constructing abstractions so that they are safe in the sense that they over-approximate the set of true executable behaviors of the system (i.e., each executable behavior is covered by an abstract execution). Thus, when these abstract behaviors are exhaustively compared to a specification and found to be in conformance, we can be sure that the true executable system behaviors conform to the specification.

We present an AI, in an informal manner, as: a domain of abstract values, an abstraction function mapping concrete program values to abstract values, and a collection of abstract primitive operations (one for each concrete operation in the program). For example, to abstract from everything but the fact that integer variable $\mathrm{x}$ is zero or not one could use the signs AI I which only keeps track of whether an integer value is negative, equal to zero, or positive. The abstract domain is the set of tokens $\{n e g, z e r o, p o s\}$. The abstraction function maps negative numbers to neg, 0 to zero, and positive numbers to pos. Abstract versions of each of the basic operations on integers are used that respect the abstract domain values. For example, an abstract version of the addition operation for signs is:

\begin{tabular}{|c||c|c|c|}
\hline$+_{\text {abs }}$ & zero & pos & $n e g$ \\
\hline \hline zero & zero & pos & neg \\
\hline pos & pos & pos & $\{z e r o$, pos, neg $\}$ \\
\hline neg & neg & $\{z e r o$, pos, neg $\}$ & neg \\
\hline
\end{tabular}

Abstract operations are allowed to return sets of values to model lack of knowledge about specific abstract values. This imprecision is interpreted in the model checker as a nondeterministic choice over the values in the set. Such cases are a source of "extra behaviors" introduced in the abstract model due to its overapproximation of the set of behaviors of the original system.

\subsection{Property Abstraction}

When abstracting properties, Bandera uses an approach similar to 17. Informally, given an AI for a variable $\mathrm{x}$ (e.g. signs) that appears in a proposition (e.g., $x>0$ ), we convert the proposition to a disjunction of propositions of the form $\mathrm{x}==a$, where $a$ are the abstract values that correspond to values that imply the truth of the original proposition (e.g., $\mathrm{x}==$ pos implies $\mathrm{x}>0$, but $\mathrm{x}==n e g$ and $\mathrm{x}==$ zero do not; it follows that proposition $\mathrm{x}>0$ is abstracted to $\mathrm{x}==p o s$ ). Thus, this disjunction under-approximates the truth of a concrete proposition insuring that the property holds on the original program if the abstracted property holds on the abstract program. 

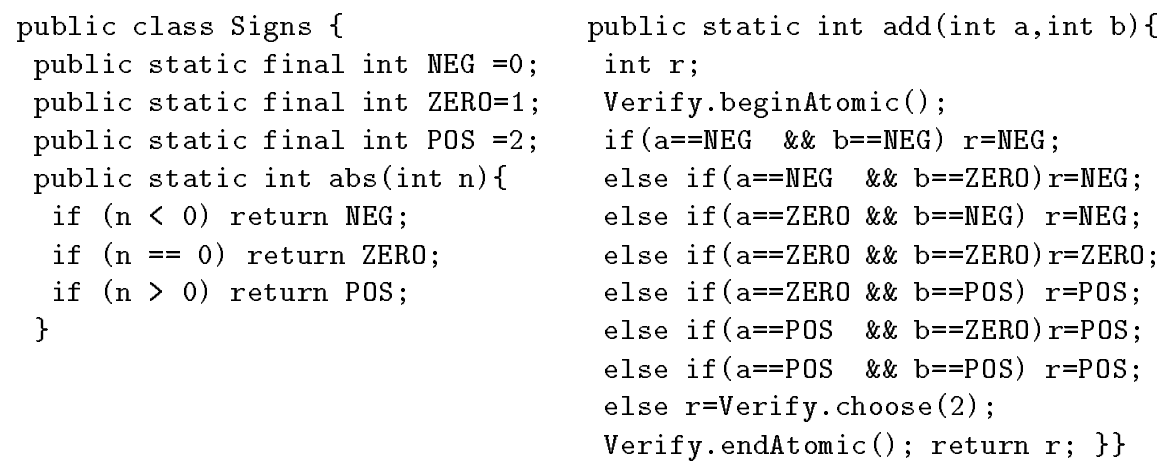

Fig. 1. Java Representation of signs AI (excerpts)

\subsection{Scheduler Abstraction}

Analyzing concurrent systems requires safe modeling of the possible scheduling decisions that are made in executing individual threads. Since software is often ported to operating system's with different scheduling policies, a property checked under a specific policy would be potentially invalid when that system is executed under a different policy. To address this, the approach taken in existing model checkers is to implement what amounts to the most general scheduling policy (i.e., nondeterministic choice among the set of runnable threads). Properties verified under such a policy will also hold under any more restrictive policy. Fairness constraints are supported in most model checkers to provide the ability to more accurately model realistic scheduling policies.

The Java language has a relatively weak specification for its thread scheduling policy. Threads are assigned priorities and a scheduler must ensure that "all threads with the top priority will eventually run" ?. Thus, a model checker that guarantees progress to all runnable threads of the highest priority will produce only feasible schedules; JPF implements this policy.

\subsection{Abstraction Implementation}

In Bandera, generating an abstract program involves the following steps: the user selects a set of AIs for a program's data components, then type inference is used to calculate the abstractions for the remaining program data, then the Java class that implements each AI's abstraction function and abstract operations is retrieved from Bandera's abstraction library, and finally the concrete Java program is traversed, and concrete literals and operations are replaced with calls to classes that implement the corresponding abstract literals and operations.

Figure II shows excerpts of the Java representation of the signs AI. Abstract tokens are implemented as integer values, and the abstraction function and operations have straightforward implementations as Java methods. For Java basetypes, the definitions of abstract operations are automatically generated using a theorem prover (see 13 for details). Nondeterministic choice is specified by 


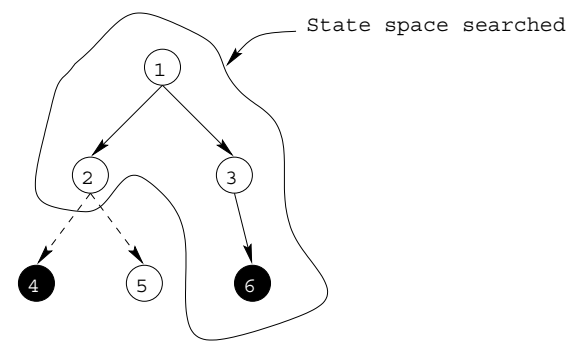

Fig. 2. Model Checking on Choose-free Paths

calls to Verify.choose(n), which JPF traps during model checking and returns nondeterministic values between 0 and $\mathrm{n}$ inclusive. Abstract operations execute atomically (via calls to Verify.beginAtomic() and Verify . endAtomic()) since they abstract concrete byte-codes (e.g. Signs.add() abstracts iadd).

\section{Finding Feasible Counter-examples}

We have seen in the previous section that, if a specification is true for the abstracted program, it will also be true for the concrete program. However, if the specification is false for the abstracted program, the counter-example may be the result of some behavior in the abstracted program which is not present in the original program. It takes deep insight to decide if an abstract counter-example is feasible (i.e. corresponds to a concrete computation). We have developed two techniques that automate tests for counter-example feasibility: model checking on choose-free paths and abstract counter-example guided concrete simulation.

\subsection{Choose-Free State Space Search}

We enhanced the JPF model checker with an option to look only at paths that do not refer to instructions that introduce nondeterminism (i.e. a Verify.choose() call). When a choose occurs the search algorithm of the model checker backtracks. The approach exploits the following theorem from 13 : Theorem.

Every path in the abstracted program where all assignments are deterministic is a path in the concrete program.

In 23 , the theorem is used to judge a counter-example feasible, whereas we use it to bias the model checker to search for feasible counter-examples. The theorem ensures that paths that are free of nondeterminism correspond to paths in the concrete program (a more general definition of deterministic paths can be found in 10 ). It follows that if a counter-example is reported in a choose-free search then it represents a feasible execution. If this execution also violates the property, then it represents a feasible counter-example.

Consider an abstracted program (whose state space is sketched in Figure . Black circles represent states where some assertion is violated. Dashed lines 


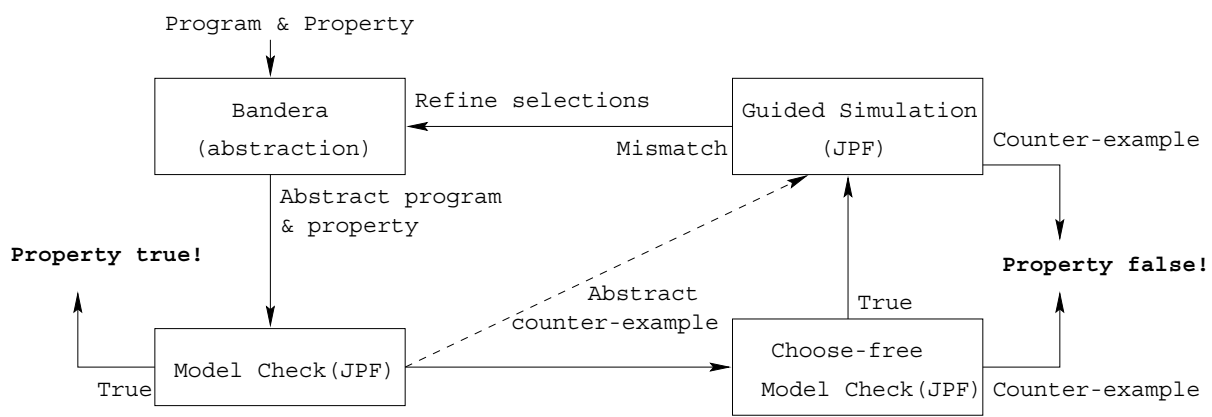

Fig. 4. Model Checking and Refinement

byte-code, there is a set of "abstract" byte-codes that execute atomically in JPF. This property of Bandera abstraction, together with the fact that all Java variables have known initial values, allows for simulation of the concrete program, based on an abstract counter-example.

This is done in JPF by executing the steps in the abstract trace. For clarity, we'll discuss the simulation in terms of the execution of lines of Java source code, but JPF can also perform simulation on a byte-code level. Each step contains information about the thread to be run next and the line of the counter-example. At each step of the concrete execution, JPF checks that the concrete line to be executed corresponds to the abstract line in the counter-example. If the lines match throughout the simulation then the abstract trace is feasible, otherwise, the abstract trace is spurious. To check whether the feasible trace is a counterexample, we have also to check if it violates the property.

Consider again the example from Figure 3 where the result of model checking the abstracted program is a counter-example where Global. done is set true after the loop in the main thread is executed two times. This means that the assertion is reachable (and violated) by the (abstract) trace

$1-2-3-4-5-3-4-5-3-4$

in the main thread. While this is clearly possible in the abstract program (since, after the abstract value of $i$ becomes pos, the condition at line 3 can be nondeterministically true or false), it is not possible in the concrete program. To see this, we simulate the steps from the abstract trace on the concrete program: after executing the loop two times, the value of $i$ is 2 so the exit condition of the loop is true and the loop is exited. At this point a line mismatch is detected and the simulation stops.

It is possible to detect the infeasibility of an abstract trace earlier, using a technique similar to forward analysis (e.g. \%): when simulating each step on the concrete program, we also check the correspondence between concrete and abstract values. This can be done in JPF by abstracting the values of variables (e.g., via calls to Signs.abs ()) in the concrete simulation and comparing them to the abstract values in the counter-example. 
[1] $\mathrm{x}=1$;

[2] $y=x+1$;

[3] assert $(x<y)$;

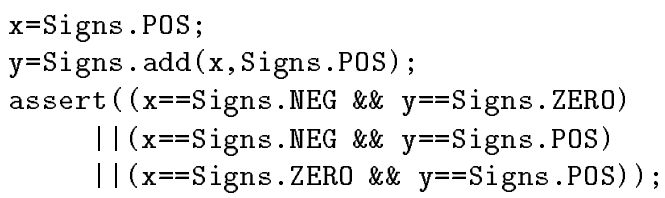

Fig. 5. Example of Spurious Error Introduced by Property Abstraction

\subsection{Methodology}

Our methodology for model checking and abstraction involves the integration of the above two techniques as illustrated in Figure 4 The input (concrete) program and the specification are abstracted (using abstractions from Bandera's library) as described in Section 2 and the transformed program is fed to a model checker. If the result of model checking is true, then the specification is true for the concrete program. If the result is false, we re-run the model checker to search only choose-free paths in the model. If the model checker finds a choose-free counter-example, it is reported to the user otherwise we perform counter-example guided simulation. If the simulation succeeds, a counter-example is reported, but if a mismatch is detected then abstractions need to be refined. The refinement involves modifying the selection of abstractions guided by the counter-example reported in the first run of the model checker. For a discussion on how the abstractions could be refined, see Section 6

\subsection{Discussion}

In general, the result of model checking an abstract program is false either because the concrete program does not satisfy the property (in which case the counter-example is feasible and indicates a real defect), or because the abstraction is not suitable for checking the property. In the latter case, the abstract counter-example can be one of the following:

- not feasible as a result of over-approximation of the behavior of the concrete program (e.g. the spurious counter-example of the program in Figure $\mathbf{3}$;

- feasible but not defective; as a result of the under-approximation of the property to be checked. This case is illustrated by the code in Figure $\mathbf{5}$ where both $\mathrm{x}$ and $\mathrm{y}$ are abstracted to signs. The predicate in the assertion is abstracted in such a way that if the assertion is true in the abstracted program, it follows that it is true in the concrete program. Abstract trace 1-2-3 violates the assertion, since after step 2, both $\mathrm{x}$ and $\mathrm{y}$ are pos. However, in the concrete program, the assertion is true.

In our experience this second case is rare, since in Bandera user's are guided to make abstraction selections that are able to decide both the truth and falsity of the propositions used in the property to be checked. Only when such a selection is impossible can a feasible, but not defective, counter-example arise.

We note that both choose-free model checking and abstract counter-example guided concrete simulation can be directly applied to a executable program slice. 
If a trace is feasible in the sliced program, it is also feasible in the original program 15. We also note that the techniques presented here can be applied for checking safety properties expressed in any universal temporal logic.

\section{$5 \quad$ Experience with Defective Java Applications}

To illustrate the potential benefits of the techniques described in the previous section, we applied them to several small to medium-size multi-threaded Java applications. These applications used both lock synchronization and conditionbased synchronization (i.e., wait/notify). The systems are: RAX (Remote Agent experiment) 2. , a Java version of a component extracted from an embedded spacecraft-control application, Pipeline $\mathbf{7}$, a generic framework for implementing multi-threaded staged calculations, RWVSN, Lea's $\mid \boldsymbol{\gamma}$ generic readers-writers synchronization framework, and DEOS 2I 2.5, the scheduler from a real-time executive for avionics systems that was translated from $\mathrm{C}++$. The following table gives some basic measures of the size of the system; SLOC stands for the number of source lines of code.

\begin{tabular}{||r|c|c|c|c|c||}
\hline \hline Program & SLOC & Classes & Methods & Fields & Threads \\
\hline \hline RAX & 55 & 4 & 8 & 7 & 3 \\
\hline Pipeline & 103 & 5 & 10 & 7 & 5 \\
\hline RWVSN & 590 & 5 & 43 & 10 & 5 \\
\hline DEOS & 1443 & 20 & 91 & 92 & 6 \\
\hline \hline
\end{tabular}

Most of these programs use the basic features of Java and its concurrency constructs, however, the RWVSN application uses abstract classes, inheritance, and java.util.vector.

The RAX and DEOS examples had known errors that we checked for. For the Pipeline and RWVSN examples we seeded faults in the program. For example, we dropped a negation (!) in one program and changed < = into < (simulating an off-by-one error) in the other. It is interesting to note that not all seeded faults could be detected given the properties we checked for, so we altered the faults until we generated a property violation.

We now describe several model checks for these systems and the automated analysis of the resulting counter-examples. Full details for the examples and model checks is available at I ittp://www.cis.ksu.edu/ pcorina/case-studies-.

\subsection{Description of Experiments}

We model checked the RAX example to detect deadlocks using two different abstractions. Figure 6 shows excerpts from the original and the generated abstract Java program. The abstraction of the program was driven by our selection that the Event.count field should be abstracted with signs. Bandera's abstraction type inference determined that the local count variables in the FirstTask.run() method should also be abstracted. Running JPF on this abstracted system detects a deadlock and produces a 74 step counter-example. 

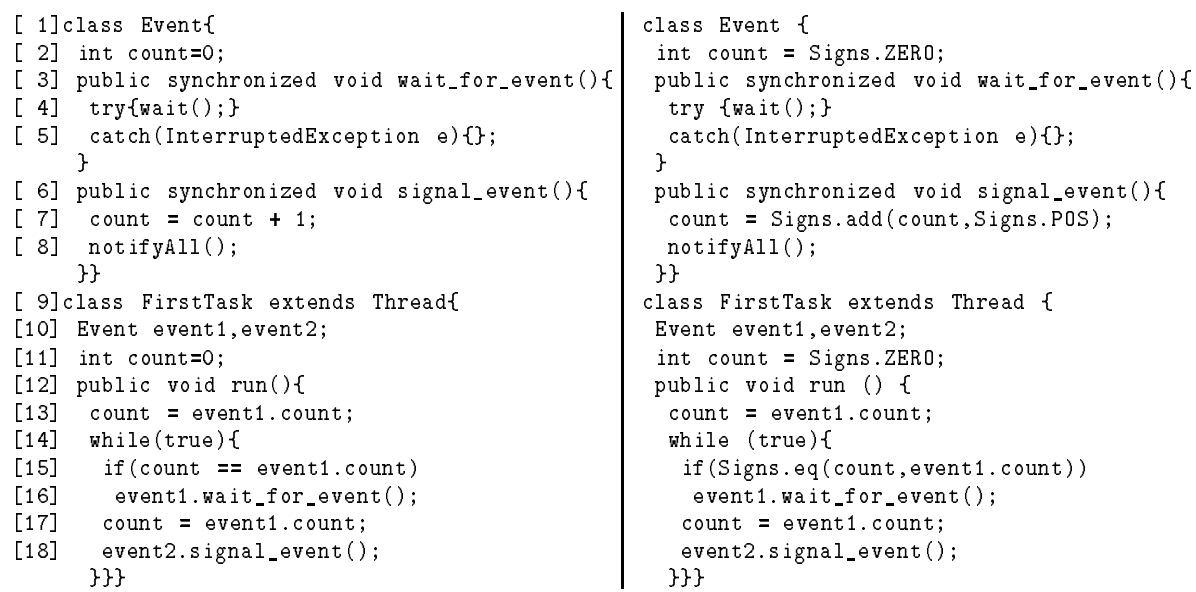

Fig. 6. RAX Program with Deadlock (excerpts)

Analysis of this counter-example reveals that it is spurious. After 39 steps in the counter-example the trace reaches the conditional at line 15 . In the real system, the branch condition is false, but due to the nondeterminism of Signs.eq() for positive parameters the abstract system enters the conditional. JPF is able to find a 40 step choose-free counter-example. It is clear that the presence of spurious counter-examples is closely related to the property being checked, the program and the abstraction's selected. We reran our model checks changing the abstraction for Event.count field to record information about the evenness or oddness of its values. This produced a 128 step counter-example, but JPF was unable to find a choose-free counter-example. At this point, we ran JPF in simulation mode guided by the 128 step counter-example and while this counterexample did contain nondeterministic choices it was shown to be feasible.

The Pipeline example consists of an application that uses the methods of a Pipeline class to manage execution of a multi-threaded staged computation. The application constructs and starts execution of a pipeline, calls stop() to end execution of the pipeline, and calls add() to provide input to the computation. We model checked a precedence property for the Pipeline system stating that "no pipeline stage (i.e., thread) will terminate until the stop method is called". Since JPF does not currently support checking of temporal properties, we encoded this using a boolean variable, stopCalled, set to true when the stop() method had been called and embedded assert (stopCalled) at the return point of the stage run methods. This example was abstracted by identifying a loop index variable that controlled the number of times the add() method was called and abstracting it to signs. Type inference determined that 5 additional fields and local variables also needed abstraction. Checking the property on the abstracted system detected an error on a 168 step counter-example. JPF found a 69 step choose-free counter-example that is similar to the example in Figure in that it occurred on the first iteration of an abstracted loop. 
RWVSN consists of an application that extends Lea's RWVSN class 18 to implement an object with a readers-writers synchronization policy. That object is then shared by several threads that read and write through the RWVSN interface. We checked that access by a reader excluded access by a writer by setting a boolean variable, in_writer, in the writer's critical section and resetting it upon exit, and embedding assert (! in_writer) in the reader's critical section. Abstraction was applied to 3 integer fields of the RWVSN class abstracting them to signs. Checking the property on the abstracted system detected an error in 179 steps. JPF found a 76 step choose-free counter-example.

The DEOS system has been the subject of several recent case studies in model checking code 21 2513 ; we performed the abstraction and analysis as described in 13. The property being checked is an assertion that encodes a test for time partitioning in the scheduler component of the system. We used dependence analysis driven by the location of the assert statement and the data values it referenced to identify a single field (out of 92) as influencing the property. We selected the signs AI for that field and type inference determined that 2 more fields should be abstracted. Checking the property on the abstracted system detected an error in 471 steps. JPF found a 312 step choose-free counter-example.

\subsection{Discussion}

While these programs represent a range of different patterns of concurrency (e.g., clients and server, pipelines, and peer-groups) and the larger examples are real applications, we do not claim that our results generalize to a broader class of multi-threaded Java programs. We do, however, believe the results suggest that the counter-example analysis techniques we have developed have merit and can significantly reduce the burden users face when analyzing counter-examples from checks of abstracted systems.

The data clearly show that counter-examples can be reduced significantly in length; this alone makes it easier to diagnose the program fault. The fact that counter-examples are guaranteed to be feasible helps focus the user's attention on only those counter-examples for which analysis will lead to fault detection.

It should come as no surprise that a choose-free model check is faster than a typical model check since it is essentially a depth-bounded model check. Most model checkers can do depth-bounded search and in fact this often allows for detection of significantly shorter counter-examples. The key difference lies in the fact that a choose-free search uses an adaptive depth-bound that is based on encountering nondeterministic choice operators. This guarantee of not executing a choice operator is what assures counter-example feasibility. Without that a naive depth-bounded search may include execution of a choice operator.

Finally, we observe that choose-free search can be an effective way to exploit more aggressive abstraction approaches. The application of source-level predicate abstraction techniques to the DEOS and RAX is described in detail in 25. In that work a predicate abstraction and an invariant for DEOS and 4 different predicate abstractions for $\mathbf{R A X}$ were used to produce abstract models that preserved both truth and falsity of the properties being checked. In contrast, 
the checks described in this paper sacrifice precision for more aggressive abstraction, and state-space reduction, while choose-free search enables the recovery of feasible counter-examples.

\section{$6 \quad$ Related and Future Work}

In our previous work 1.3, we focused on the specification, generation, selection and compilation of abstractions for Java programs. In this paper, we detail techniques for analyzing counter-examples and provide evidence for their usefulness on several non-trivial Java programs.

Most existing work on counter-example analysis is oriented towards the goal of verification; counter-example analysis drives abstraction refinement for the purpose of proving a property. In contrast, our work is oriented toward defect detection. Our biasing of the model checker yields a complete coverage of the sub-space of guaranteed feasible paths in the system rather than simply assessing the feasibility of a single counter-example from an unbiased model check.

Our simulation technique works because JPF maintains a correspondence between the concrete and abstracted programs and Java defines default initial values for all data (thus a program has a single initial state). It is possible to develop more general simulation techniques that handle multiple initial states, but we believe these are not necessary for Java. One such technique 5 uses forward analysis and performs a symbolic simulation of the concrete system using predicates that characterize the program data values. Since it does not keep a correspondence between concrete and abstract transitions, rather than determine the next concrete state it must compute (at each step of the simulation) the set of all possible next concrete states. This method, which is implemented in SMV, is limited to finite-state systems.

In SLAM 3, sequential C programs are abstracted into boolean programs; symbolic execution is used to map abstract counter-examples to concrete executions. INVEST 4 and interactive abstractions $\%$ use theorem proving to rule out spurious counter-examples. Backward analysis is used to obtain information to refine the abstractions. Unlike our approach, these tools/techniques are not concerned with property abstraction or scheduling information.

We believe that the methods described in these papers are complementary to our techniques. For example, we can use backward analysis to obtain feedback for refinement of abstractions. Backward analysis computes pre-images of the violating abstract state over the given trace. For the spurious counter-example of Figure 3 after the body of the loop is executed two times, the value of the loop condition is true, which means that the concrete value of $\mathrm{x}$ is believed to be less than 2. The analysis would discover that this happens because the value of $\mathrm{x}$ before the assignment at line 5 is believed to be less than 1 (which is not true in the concrete program, where the value of $\mathrm{x}$ is exactly 1 ). This implies that a new abstraction to be selected for variable $\mathrm{x}$ has to include a new token for 1 (e.g. signs abstraction should be replaced with range(0..1) abstraction 13). 
We note that both choose-free search and counter-example guided simulation techniques could be implemented in any explicit-state model checker. For example, Bandera 8 generates Promela models for Spin that can easily be adapted to perform choose-free search. Path simulation simply requires the ability to associate the steps of the concrete and abstract program and to simulate the concrete program. One can already do this by hand using Spin's simulation facilities, but automating the process would greatly ease its use. We also note that, although we set our presentation in the context of Bandera's abstraction, other forms of data abstraction, like JPF's predicate abstraction, would also be treated properly. By that we mean that a path through the predicate abstracted code that is choose-free or that can be mapped to a concrete execution is feasible.

\section{Conclusion}

In this paper, we have suggested two approaches for analyzing counter-examples produced by model checks of abstracted programs. These approaches have the advantage of being very fast (i.e., choose-free search is depth-bounded and the cost of simulation is related to the length of the counter-example). Based on experimentation with an implementation of these techniques in a Java model checking tool we have also found the techniques to be capable of detecting guaranteed feasible counter-examples in nearly every case. This enables aggressive abstractions to be applied without losing the ability to detect errors, thereby minimizing the need for refinement of abstractions. This implementation treats not only abstraction of program data, but also of thread scheduling policies, and the property to be checked. Finally, we believe that these techniques can be combined with other counter-example analysis methods to provide a suite of tools that vary cost and in their ability to precisely analyze counter-examples. Such a tool suite would be a useful addition to any model checking tool.

\section{References}

1. S. Abramsky and C. Hankin. Abstract Interpretation of Declarative Languages. Ellis Horwood Limited, 1987.

2. K. Arnold and J. Gosling. The Java Programming Language. Addison-Wesley, 1998.

3. T. Ball and S.K. Rajamani. Checking temporal properties of software with boolean programs. In Proc. of the Workshop on Advances in Verification, July 2000.

4. S. Bensalem, Y. Lakhnech, and S. Owre. Computing abstractions of infinite state systems compositionally and automatically. In Proc. 10th International Conference on Computer-Aided Verification, June 1998.

5. E.M. Clarke, O. Grumberg, S. Jha, Y. Lu, and H. Veith. Counterexample-guided abstraction refinement. In Proc. 12 th International Conference on Computer-Aided Verification, July 2000.

6. E.M. Clarke, O. Grumberg, and D.E. Long. Model checking and abstraction. ACM Transactions on Programming Languages and Systems, 16(5):1512-1542, September 1994. 
7. J.C. Corbett, M. B. Dwyer, J. Hatcliff, and Robby. Bandera : A source-level interface for model checking Java programs. In 14.

8. J.C. Corbett, M.B. Dwyer, J. Hatcliff, S. Laubach, C.S. Păsăreanu, Robby, and H. Zheng. Bandera : Extracting finite-state models from Java source code. In 14.

9. P. Cousot and R. Cousot. Abstract interpretation: A unified lattice model for static analysis of programs by construction or approximation of fixpoints. In Conference Record of the Fourth Annual ACM Symposium on Principles of Programming Languages, pages 238-252, 1977 .

10. D. Dams, R. Gerth, G. Dhmen, R. Herrmann, P. Kelb, and H. Pargmann. Model checking using adaptive state and data abstraction. In D.L.Dill, editor, Proc. 6th International Conference on Computer-Aided Verification, volume 818 of Lecture Notes in Computer Science, pages 455-467. Springer Verlag, June 1994.

11. D. Dams, R. Gerth, and O. Grumberg. Abstract interpretation of reactive systems. ACM Transactions on Programming Languages and Systems, 19(2):253-291, March 1997.

12. C. Demartini, R. Iosif, and R. Sisto. A deadlock detection tool for concurrent Java programs. Software - Practice and Experience, 29(7):577-603, July 1999.

13. M.B. Dwyer, J. Hatcliff, R. Joehanes, S. Laubach, C.S. Păsăreanu, Robby, W. Visser, and H. Zheng. Tool-supported program abstraction for finite-state verification. In Proceedings of the 23rd International Conference on Software Engineering, May 2001.

14. C. Ghezzi, M. Jazayeri, and A. Wolf, editors. Proceedings of the 22nd International Conference on Software Engineering, June 2000.

15. J. Hatcliff, J.C. Corbett, M.B. Dwyer, S. Sokolowski, and H. Zheng. A formal study of slicing for multi-threaded programs with JVM concurrency primitives. In Proceedings of the 6th International Static Analysis Symposium, September 1999.

16. G.J. Holzmann. The model checker SPIN. IEEE Transactions on Software Engineering, 23(5):279-294, May 1997.

17. Y. Kesten and A. Pnueli. Modularization and abstraction: The keys to practical formal verification. Lecture Notes in Computer Science, 1450, 1998.

18. D. Lea. Concurrent Programming in Java[tm], Second Edition: Design principles and Patterns. The Java Series. Addison-Wesley, 2nd edition, 1999.

19. C. Loiseaux, S. Graf, J. Sifakis, A. Bouajiani, and S. Bensalem. Property preserving abstractions for the verification of concurrent systems. Formal Methods in System Design, 6(1):11-44, 1995.

20. K.L. McMillan. Symbolic Model Checking. Kluwer Academic Publishers, 1993.

21. J. Penix, W. Visser, E. Engstrom, A. Larson, and N. Weininger. Verification of time partitioning in the DEOS real-time scheduling kernel. In 14.

22. V. Rusu and E. Singerman. On proving safety properties by integrating static analysis, theorem proving and abstraction. In Proceedings of Tools and Algorithms for the Construction and Analysis of Systems, March 1999.

23. H. Saïdi. Model checking guided abstraction and analysis. In Proceedings of the 7th International Static Analysis Symposium, 2000.

24. W. Visser, G. Brat, K. Havelund, and S. Park. Model checking programs. In Proceedings of the 15th IEEE International Conference on Automated Software Engineering, September 2000.

25. W. Visser, S. Park, and J. Penix. Applying predicate abstraction to model check object-oriented programs. In Proceedings of the 3rd ACM SIGSOFT Workshop on Formal Methods in Software Practice, August 2000. 